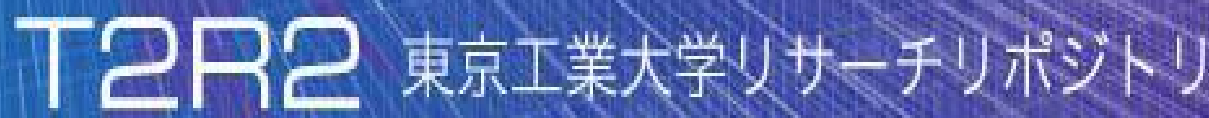

\section{Tokyo Tech Research Repository}

\section{論文 /著書情報 \\ Article /Book Information}

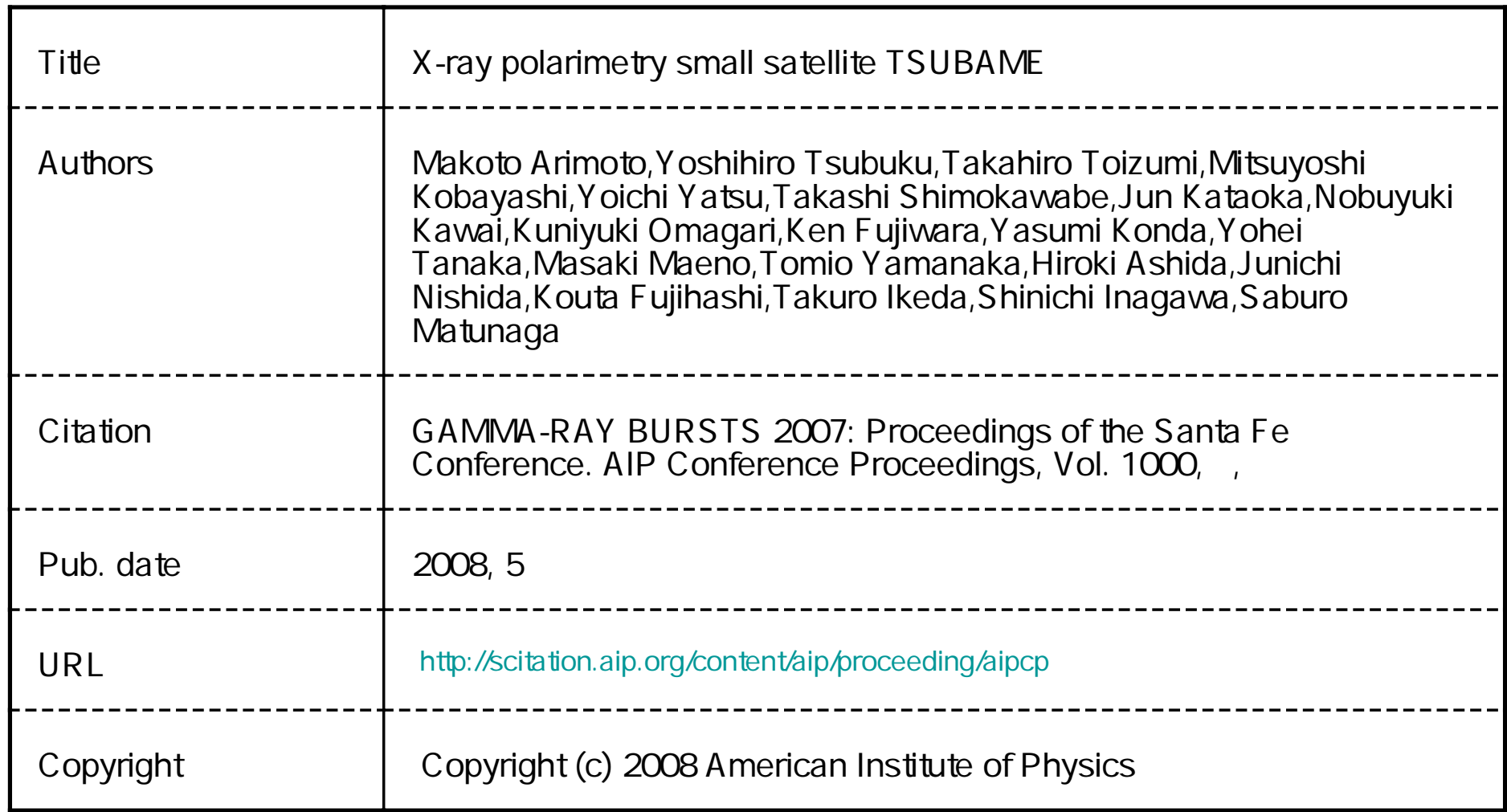




\title{
X-ray polarimetry small satellite TSUBAME
}

\author{
Makoto Arimoto*, Yoshihiro Tsubuku*, Takahiro Toizumi*, Mitsuyoshi \\ Kobayashi*, Yoichi Yatsu*, Takashi Shimokawabe*, Jun Kataoka*, \\ Nobuyuki Kawai*, Kuniyuki Omagari ${ }^{\dagger}$, Ken Fujiwara ${ }^{\dagger}$, Yasumi Konda ${ }^{\dagger}$, \\ Yohei Tanaka ${ }^{\dagger}$, Masaki Maeno ${ }^{\dagger}$, Tomio Yamanaka ${ }^{\dagger}$, Hiroki Ashida ${ }^{\dagger}$, \\ Junichi Nishida ${ }^{\dagger}$, Kouta Fujihashi ${ }^{\dagger}$, Takuro Ikeda ${ }^{\dagger}$, Shinichi Inagawa ${ }^{\dagger}$, \\ Yoshiyuki Miura ${ }^{\dagger}$ and Saburo Matunaga ${ }^{\dagger}$ \\ *Department of Physics, Tokyo Institute of Technology, 2-12-1 Ookayama, Meguro-ku, Tokyo \\ $152-8551$ \\ ${ }^{\dagger}$ Department of Mechanical and Aerospace Engineering, Tokyo Institute of Technology, 2-12-1 \\ Ookayama, Meguro-ku, Tokyo 152-8551
}

\begin{abstract}
TSUBAME" is a university-built small satellite mission to measure polarization of hard X-ray photons (30-100keV) from Gamma-ray bursts (GRBs) using azimuthal angle anisotropy of Compton-scattered photons. Polarimetry in the hard X-ray and soft $\gamma$-ray band plays a crucial role in the understanding of high energy emission mechanisms and the distribution of magnetic fields and radiation fields. TSUBAME has two instruments: the Wide-field Bust Monitor (WBM) and the Hard X-ray Compton Polarimeter (HXCP). The WBM determines on board the direction of the burst occurrence with an accuracy of 10 degrees, then using a high speed attitude control device, the HXCP is pointed to the GRB within 10 seconds after the burst occurrence to promptly detect polarized X-ray photons from the GRB.

We present a TSUBAME mission overview, results of a Monte Carlo simulation of the X-ray polarization measurement and the plans for the future of this mission.
\end{abstract}

Keywords: gamma-rays: bursts — Polarimetry

PACS: $98.70 . \mathrm{Rz}, 95.75 . \mathrm{Hi}$

\section{INTRODUCTION}

In the study of GRBs and other X-ray and $\gamma$-ray objects, the analysis of their spectrum and time variability is commonly used. But the information extracted from these observations could not yet identify the dominant emission mechanism. Measurements of the polarization can clarify the emission mechanism and the orientation of the polarization plane will provide an idea of the distribution of magnetic field, radiation field and matter around the sources (e.g. rotation powered pulsars, accreting black holes and active galactic nuclei; AGNs). The reliable polarimetry in soft X-ray band has been reported at the energies 2.6 and $5.2 \mathrm{keV}$ using Bragg reflection [1]. For GRBs, only a few observations of X-ray polarimetry has been reported [2,3], and these results were disputable because of a low level of significance and large systematic uncertainties. In such situations, new observatories of X-ray polarimetry for GRBs with high sensitivity and good calibration have been long-awaited. 


\section{TSUBAME MISSION OVERVIEW}

"TSUBAME", meaning a bird "swallow" in Japanese, is a small satellite that will measure the polarization of astronomical objects. The dimensions of TSUBAME are 50 $\mathrm{cm} \times 50 \mathrm{~cm} \times 40 \mathrm{~cm}$ and a total mass of $\sim 40 \mathrm{~kg}$. TSUBAME is planned to be launched in 2009 or later as a piggyback on H-II A rocket. The main target of TSUBAME is GRBs. A rapid observation in the study of GRBs is needed because the duration of prompt emission is generally short $(2-100 \mathrm{~s})$. A schematic observation sequence is shown in Fig. 1. When a GRB occurs, TSUBAME localizes the coordinate of the GRB on board by the localization detector Wide-field Burst Monitor (WBM). After whirling and repointing itself to the GRB with control momentum gyro (CMG) promptly $(\sim 90$ deg within $10 \mathrm{~s}$ ), the Hard X-ray Compton Polarimeter (HXCP) starts the observation within $10 \mathrm{~s}$ after the burst detection with the space craft slowly rotated around the axis to remove the systematics.

The WBM consists of 5 sets of a CsI scintillator connected to an avalanche photo diode (APD). These CsI scintillators are attached to each corner of the observatory, shown in Fig. 2. Using the count rates of 5 detectors, the localization of GRBs will be done with an accuracy of $\sim 10$ degrees by the centroid method. The field of view of the WBM is $2 \pi$ sr (half of the whole sky).

The polarimeter HXCP will measure the polarized photons in the $20-100 \mathrm{keV}$ band using the azimuthal angle anisotropy of Compton scattered photons, and consists of $8 \times$ 8 sets of a plastic scintillator (scatterer) connected to a multi-anode photomultiplier tube (MAPMT) R8900-M16-UBA ( $4 \times 4$ channels, physical dimensions $\sim 26 \times 26 \mathrm{~mm}^{2}$ and an ultra bi-alkali photo-cathode) and 36 sets of a CsI scintillator (absorber) connected to an APD S8664-55 $\left(5 \times 5 \mathrm{~mm}^{2}\right)$ [4] surrounding the $8 \times 8$ scatterers, shown in Fig. 2 . The effective area is $\sim 3.7 \mathrm{~cm}^{2}$ and its field of view is $\sim 25 \mathrm{deg}^{2}$.

The CMG is one of the momentum exchange devices, which could attain large maximum angular momentum, allowing TSUBAME to change the attitude $\sim 90 \mathrm{deg}$ within $10 \mathrm{~s}$ and do the prompt polarization observations.

Furthermore, TSUBAME aims to detect polarization from not only GRBs but also bright galactic diffuse objects (e.g., Crab Nebula), binary systems (e.g., Cyg X-1) and flares from blazars or soft $\gamma$-ray repeaters (SGRs). TSUBAME's field of view is large and has the high background rate, but if the objects are very bright ( $>1 \mathrm{Crab}$ ), TSUBAME can detect the polarization from these objects significantly.

\section{SIMULATION OF POLARIZATION MEASUREMENTS}

For the polarimeter HXCP, a simulation of Compton scattering using Geant 4 is needed to estimate the signal and background levels and the detectability of the polarization from GRBs. We simulate a GRB event; GRB 021206 which had a flux of $3.2 \times 10^{-5}$ $\mathrm{ergs} / \mathrm{cm}^{2} / \mathrm{s}$ and a duration of $5.5 \mathrm{~s}$. This GRB was very bright and its polarization value was reported, but this result was disputable [2] described in the introduction. 


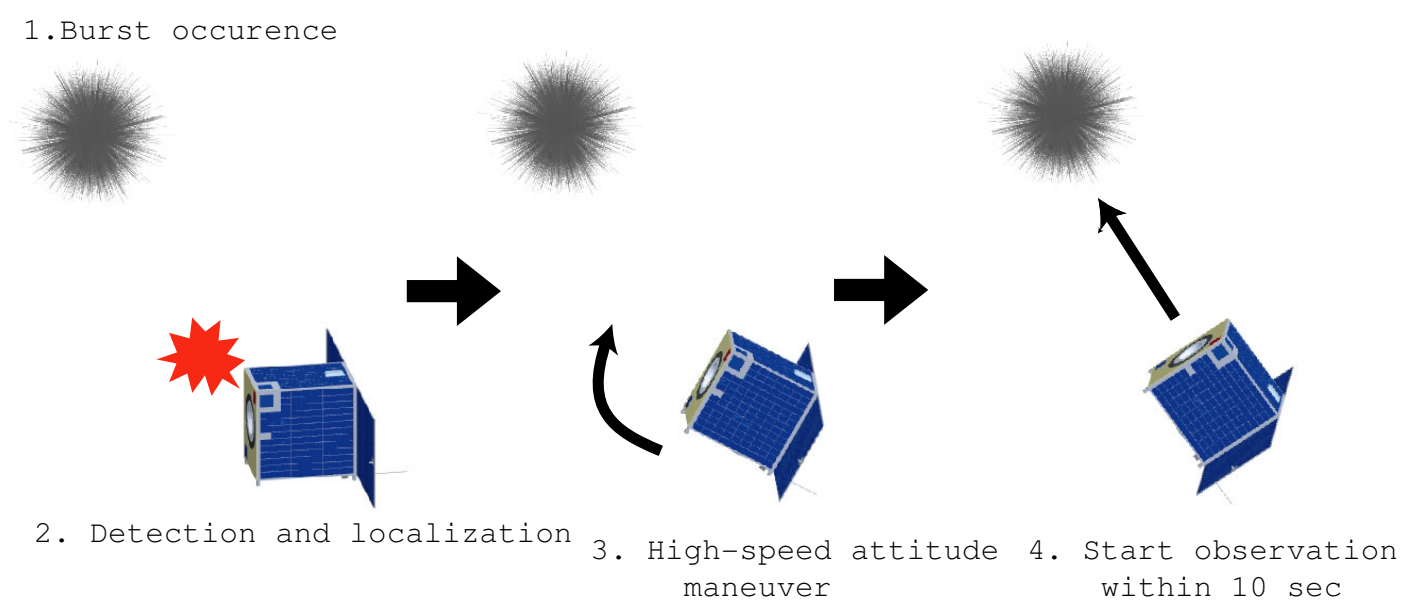

FIGURE 1. TSUBAME observation sequence

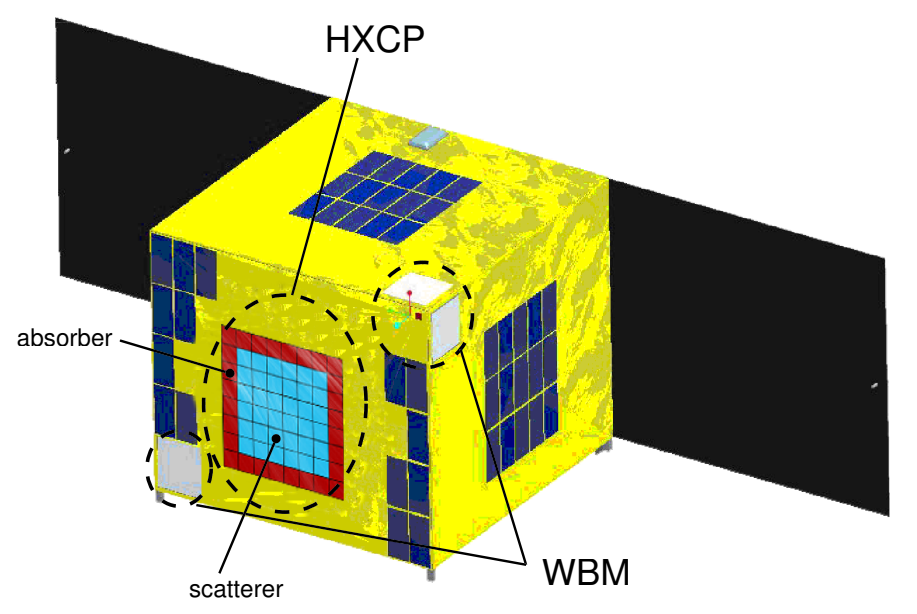

FIGURE 2. TSUBAME configuration: Hard X-ray Compton Polarimeter (HXCP) is centered at the observatory. The HXCP consists of $8 \times 8$ plastic scintillators as a scattering site and 36 CsI scintilltors as a photo-absorption site surrounding scatterers. CsI counters of the Wide-field Bust Monitor (WBM) are attached to each corner of the observatory.

In this simulation, we assume that the burst photons are $100 \%$ polarized. Here, the dominant $\gamma$-ray backgound are cosmic X-ray background (CXB) and atmospheric $\gamma$-ray. The $\gamma$-ray background flux models have been developed based on observational data [5] and we generate the CXB and atmospheric $\gamma$-ray to represent a satellite environment. Here, the background induced by high-energy charged particles has been determined to be negligible. This background should be eliminated by imposing that the pulse-height from GRBs lies between lower and upper discrimination levels because the charged particles deposit very large signals through the scintillators.

These simulation results are shown in Fig. 3. The detected GRB 021206 spectrum is plotted with thick solid line, the CXB with open triangles, the atmospheric $\gamma$-ray with open squares and the total background $\gamma$-rays with both open circles and solid line (Fig. 

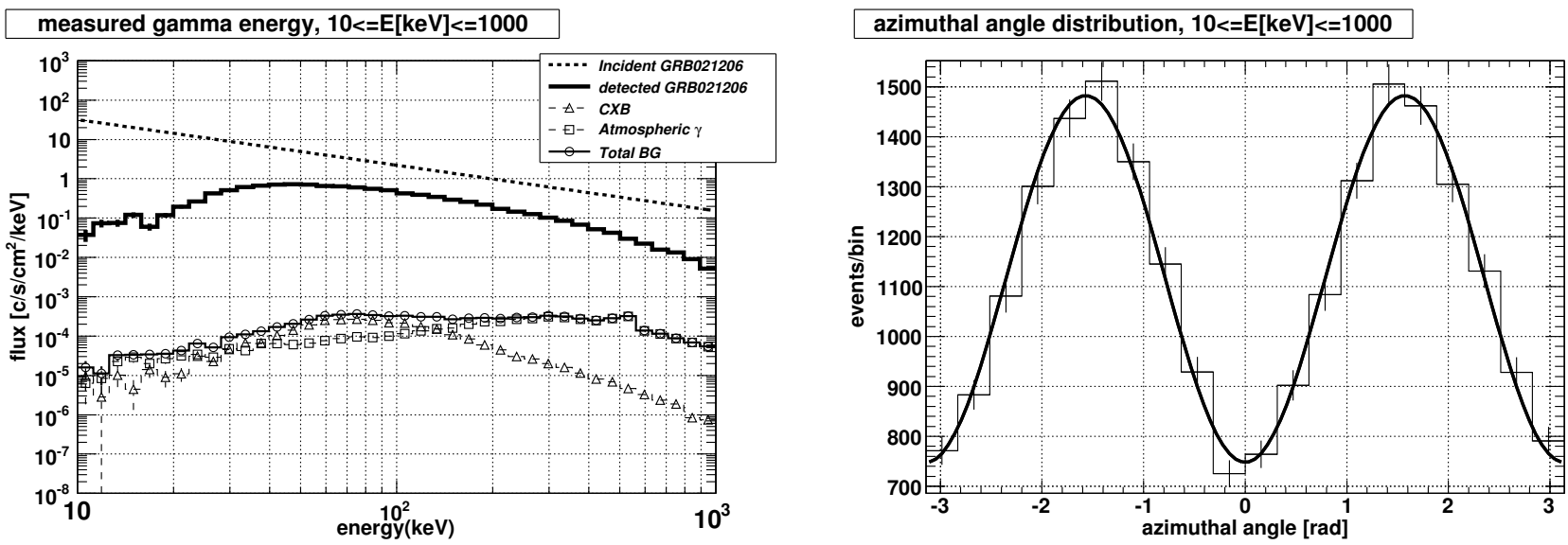

FIGURE 3. (left): The signal rate expected for GRB 021206 (thick solid line) is significantly higher than the expected background rate for $\mathrm{CXB}$ and atmospheric $\gamma$-ray (thin solid line with open squares denotes the total background). (right): Modulation curve in the azimuthal Compton scattering angle for GRB 021206. $\mathrm{MF}=33.0 \pm 0.9 \%$.

3 left). We find that the detected GRB 021206 flux is significantly higher than the total $\gamma$-ray background. Using the valid polarization events (a Compton scattered event and a photo-absorbed event), we make an azimuthal modulation curve (Fig. 3 right) and calculate the modulation factor (MF) for GRB021206 including the $\gamma$-ray backgrounds. The MF is $33.0 \pm 0.9 \%$, meaning that we will be able to detect the polarization of this burst at $\sim 33 \sigma$ confidence level. We also derived a minimum detectable polarization (MDP) of $6.3 \%$ at $3 \sigma$ confidence level, and find that TSUBAME has a higher ability to detect polarization compared with past astronomical observatories.

\section{FUTURE}

We plan to estimate the performance of the prototype of the HXCP including plastic scintillators coupled to MAPMTs and CsI scintillators coupled to APDs using a Xray beamline, and need the development of its readout electronics. For the WBM to localize GRBs' position, we first need to estimate the ability of the localization using Geant4. Then, we optimize the design of the WBM and develop its engineering model. Furthermore, prototypes of the CMG and some subsytems (radiation belt monitor and attitude control subsystem etc.) will be tested in parallel.

\section{REFERENCES}

1. M. C. Weisskopf et al., ApJL 208, L125-L128 (1976).

2. W. Coburn, and S. E. Boggs, Nature 423, 415-417 (2003).

3. S. McGlynn et al., A\&A 466, 895-904 (2007).

4. T. Ikagawa et al., NIMA 515, 671-679 (2003).

5. T. Mizuno et al., ApJ 614, 1113-1123 (2004). 